\title{
New Classes of Regular Symmetric Fractals
}

This paper was downloaded from TechRxiv (https://www.techrxiv.org).

\section{LICENSE}

CC BY 4.0

SUBMISSION DATE / POSTED DATE

01-06-2021 / 03-11-2021

CITATION

Kak, Subhash (2021): New Classes of Regular Symmetric Fractals. TechRxiv. Preprint. https://doi.org/10.36227/techrxiv.14714094.v2

$\mathrm{DOI}$

10.36227/techrxiv.14714094.v2 


\title{
New Classes of Regular Symmetric Fractals
}

\author{
Subhash Kak \\ Oklahoma State University, Stillwater \\ Email: subhash.kak@okstate.edu
}

\begin{abstract}
The paper introduces new fractal families with annular and checkerboard structures that include the Sierpinski carpet and the Menger sponge as special cases. The complementary mapping is defined and a notation to represent the families is proposed. The new classes represent an enhanced set with potential applications to natural and engineered self-similar systems.
\end{abstract}

\section{Introduction}

The importance of fractals to the study of natural, social and engineered systems is well established [1][2]. They belong to the larger field of scale-invariant and selfsimilar systems [3][4][5][6][7][8]. In a recent paper [9], novel fractals with dimension that is close to the optimal value from the perspective of information efficiency [10][11][12] were presented. Designs were found for up to ten-way branching at each iteration and they included symmetric and asymmetric ones, as well as those where in addition to holes, a few layers of other regions are peeled. Only representative fractals were described with the hope that the method can be used to find many other designs that will provide insight into systems in biology, chemistry, polymers, and the earth sciences.

In more advanced investigations, the mathematical basis of noninteger spaces [13][14] will have to be brought into the analysis. Some progress in that direction has been made in applications to the information dimension of physical space [15] which takes us to the view that space is not to be seen as a three-dimensional container. The evolutionary stages of a noninteger dimensional physical space were considered [16], and this has applications to astronomical systems as well as metamaterials [17][18].

This paper lists new fractals that are motivated by examples of physical systems where influences flow out from the center in a manner that is captured by annular mapping as well as those given by checkerboard patterns. This work has many 
parallels with constructions in [9]. Since natural and engineered systems are likely to have a range of characteristics arising from the underlying physics associated with different values of information efficiency, the examples are of more than just a theoretical interest.

\section{Iterated function systems}

Iterated Function System (IFS) fractals are created on the basis of simple plane transformations that include scaling, dislocation and the rotation of the plane axes [19][20]. Creating an IFS fractal consists of following steps:

1. Draw an initial pattern on the plane

2. Transform the initial pattern using appropriate contracting transformation

3. Combine initial and transformed patterns

4. Repeat as many times as desired

In many cases, each iteration consists of one or more affine transformations of the type (with suitable constants $(a, b, c, e, f, g)$ :

$$
\left(\begin{array}{l}
x_{n+1} \\
y_{n+1}
\end{array}\right)=\left(\begin{array}{ll}
a & b \\
c & e
\end{array}\right)\left(\begin{array}{l}
x_{n} \\
y_{n}
\end{array}\right)+\left(\begin{array}{l}
f \\
g
\end{array}\right)
$$

For example, the binary tree fractal of Figure 1 that has been drawn to 6 iterations requires the specification of the scaling factor $r$ and the angle $\theta$.
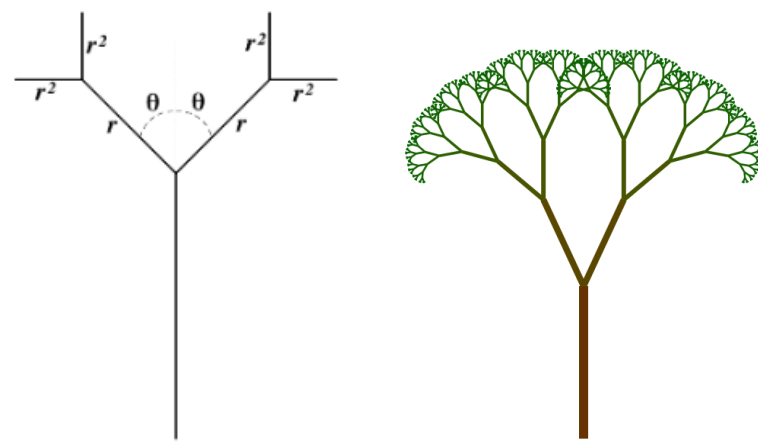

Figure 1 . The binary tree specifications and the tree with $\theta=25^{0}, r=5 / 8$

Here $a=e=r \cos \theta ; b=r \sin \theta ; c=-r ; f=0, g=1$.

We can also see the algorithm as one where each "cube" is replaced by the number $N(\epsilon)$ at each iterative step, with $\frac{1}{\varepsilon}=n$, and dimension $d$ equal to [2][9]:

$$
d=\frac{\ln N(\epsilon)}{\ln n}
$$


This provides an easy procedure to compute the dimension for regular mappings as we will see in the examples given below.

\section{Annular planar maps}

We will first consider planar fractals (dimensions $1<d<2$ ) which have annular square forms.

Definition. An annular planar fractal where at each iteration a square is replaced by $n \times n$ sub-squares with alternating bands of sides $n, n-l, n-m \ldots$ will be called $A_{n}^{2}(n, n-l, n-m, \ldots)$, where the sub-squares at the layers of $n, n-l, n-m, \ldots$ are retained and the others are removed.

Note that the layer counts of sub-squares from outside with count of $n$ in decrement by 2 .

Examples. The first iteration of two mappings based on 49 and 64 cell subdivision is presented in Figure 2.

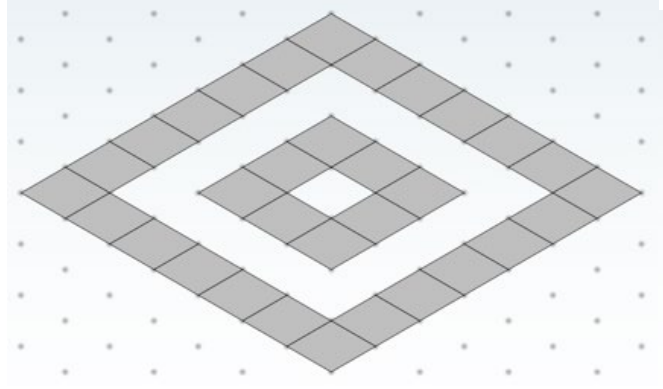

(a)

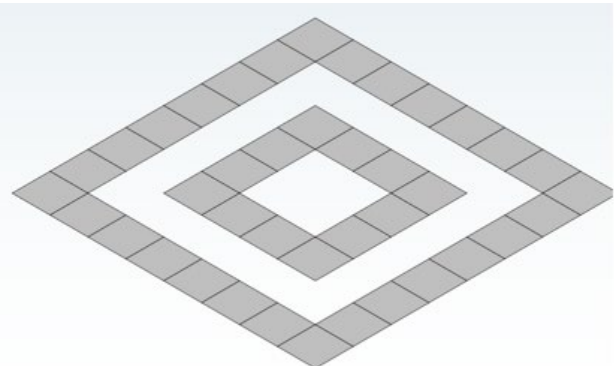

(b)

Figure 2. (a) $A_{7}^{2}(7,3)$ and (b) $A_{8}^{2}(8,4)$ fractal mappings

The left mapping shows the original square replaced by $7 \times 7$ cells of which all the ones in the outer parameter are occupied and the ones inside one sub-square removed in a square ring. Likewise, the right mapping based on $8 \times 8$ cells is $A_{8}^{2}$ $(8,4)$.

For each of these cases, we need to count the number of sub-squares that are retained in each iteration and use formula (2) to compute the dimension. In (a), the basic $7 \times 7$ plane is replaced by 25 smaller squares $=7^{2}-5^{2}+3^{2}-1=32$ and 
this substitution is repeated. In the $8 \times 8$ example of (b), each iteration gives us $8^{2}-6^{2}+4^{2}-2^{2}=(8-6)(8+6)+(4-2)(4+2)=2(14+6)=40$ subsquares. Therefore,

$$
\begin{aligned}
& d\left(A_{7}^{2}(7,3)\right)=\frac{\ln 32}{\ln 7} \cong 1.781 \\
& d\left(A_{8}^{2}(8,4)\right)=\frac{\ln 40}{\ln 8} \cong 1.774
\end{aligned}
$$

Definition. $A_{n}{ }^{\prime} s$ complementary fractal mapping where the light and dark subsquares are exchanged will be called $B_{n}$.

Definition. Let $\alpha$ represent the number of sub-squares (out of $n^{2}$ ) in the iterative map that are retained.

Corollary. $d\left(A_{n}\right)=\frac{\ln \alpha}{\ln n}$, and $d\left(B_{n}\right)=\frac{\ln \left(n^{2}-\alpha\right)}{\ln n}$

Example. The complementary mapping for $A_{7}^{2}(7,3)$ will be $B_{7}^{2}(7,3)=A_{7}^{2}(5,1)$.

$$
d\left(B_{7}^{2}(7,3)\right)=d\left(A_{7}^{2}(5,1)\right)=\frac{\ln 17}{\ln 7} \cong 1.456
$$

In Figure 3, the iteration maps each square into 81 sub-squares of which 49 are retained (shown in dark).

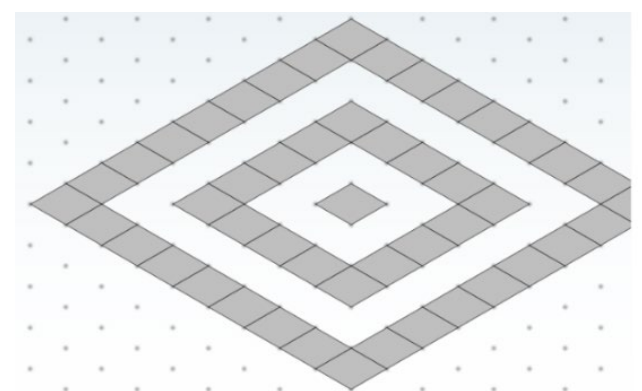

Figure 3. $A_{9}(9,5,1)$ fractal

The dimensionality of this mapping will be:

$$
d\left(A_{9}^{2}(9,5,1)\right)=\frac{\ln 49}{\ln 9} \cong 1.771
$$


For a more general case, consider the alternating annular square rings of $11 \times 11$ given in Figure 4.

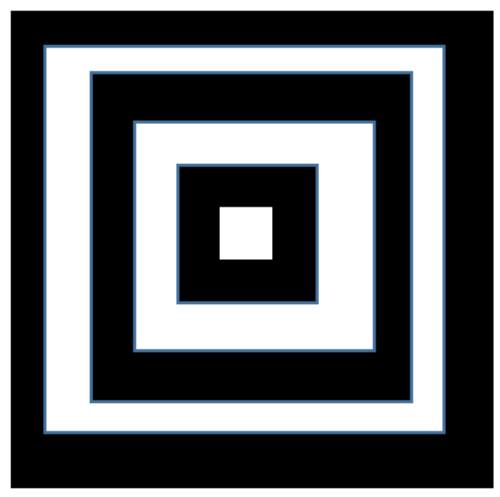

Figure 4. Alternating pattern

The sub-squares that are retained will have the count $11^{2}-9^{2}+7^{2}-5^{2}+3^{2}-$ $1=2(20+12+4)=72$. Therefore,

$$
\begin{aligned}
& d\left[A_{11}^{2}(11,7,3]=\frac{\ln 72}{\ln 11} \cong 1.784\right. \\
& 4(n-1+n-5+\cdots+n-l)
\end{aligned}
$$

where $n-l$ is the least positive term in the sequence. Simplifying, we find that for even $n$,

$$
d=\frac{\ln 0.5\left(n^{2}+2 n\right)}{\ln n}, n \text { even }
$$

For odd k,

$$
d= \begin{cases}\frac{\ln 0.5\left(n^{2}+2 n+1\right)}{\ln n} & ; n-1 \bmod 4=0 \\ \frac{\ln 0.5\left(n^{2}+2 n-1\right)}{\ln n} & ; n-1 \bmod 4 \neq 0\end{cases}
$$

These may be simplified further.

For example, (3) may be rewritten as: 


$$
d=1+\frac{\ln (0.5 n+1)}{\ln n}, n \text { even }
$$

When $\mathrm{n}=8$ (Figure $2 \mathrm{~b}$ ), $d=1+\frac{\ln 5}{\ln 8} \cong 1.774$, as computed.

Theorem. The dimensionality of the map $A$ is $s$ if $\alpha=n^{s}$.

Proof. This follows from the fact $d\left(A_{n}\right)=\frac{\ln \alpha}{\ln n}$. It also follows that $d\left(A_{n}\right)$ will be less than 1 , if $\alpha<n$, for the logarithm function is monotonically increasing.

\section{Basic planar maps}

We now consider basic annular maps for $\mathrm{n}=3,4$, and 5 as in Figure 5. The dimensionality of these maps is

$$
\begin{aligned}
& A_{3}^{2}(3), \text { also known as Sierpinski }(\text { Figures } 5 \mathrm{a} \text { and } 6)=\frac{\ln 8}{\ln 3} \cong 1.893 \\
& A_{4}^{2}(4)(\text { Figures } 5 \mathrm{~b} \text { and } 7)=\frac{\ln 12}{\ln 4} \cong 1.792 \\
& A_{5}^{2}(5,1)(\text { Figure } 5 \mathrm{c})=\frac{\ln 17}{\ln 5} \cong 1.760
\end{aligned}
$$

Their complementary maps, also shown in Figure 5, have dimensionality of $\frac{\ln 1}{\ln 3}=$ $0 ; \frac{\ln 4}{\ln 4}=1 ;$ and $\frac{\ln 8}{\ln 5} \cong 1.292$.

Clearly, the complementary maps of $A_{3}^{2}(3)$ and $A_{4}^{2}(4)$ are not interesting with values of 0 and 1 for the first one telescopes down to nothingness, whereas, the second is a stable point.

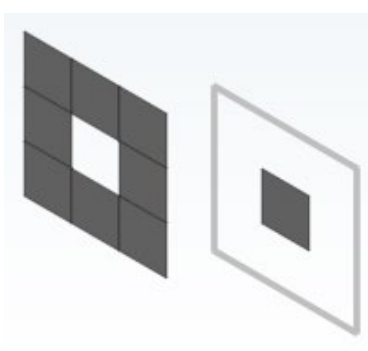

(a)
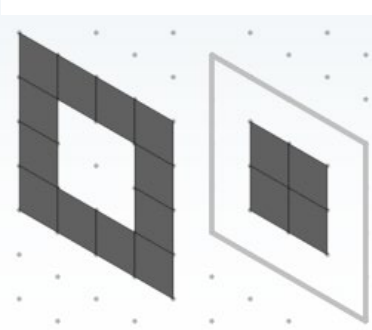

(b)

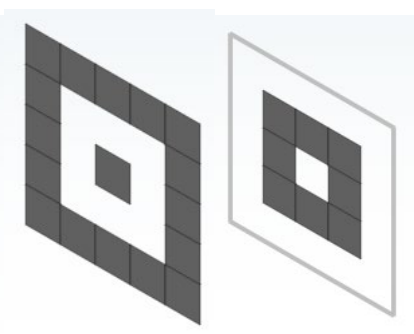

(c)

Figure 5. (a) $A_{3}^{2}(3)$, (b) $A_{4}^{2}(4)$, (c) $A_{5}^{2}(5,1)$, together with their complementary mappings 
As mentioned before, $A_{3}^{2}(3)$ is the Sierpinski map [21], which is shown below in Figure 6 for its second, third, and fourth iterations:

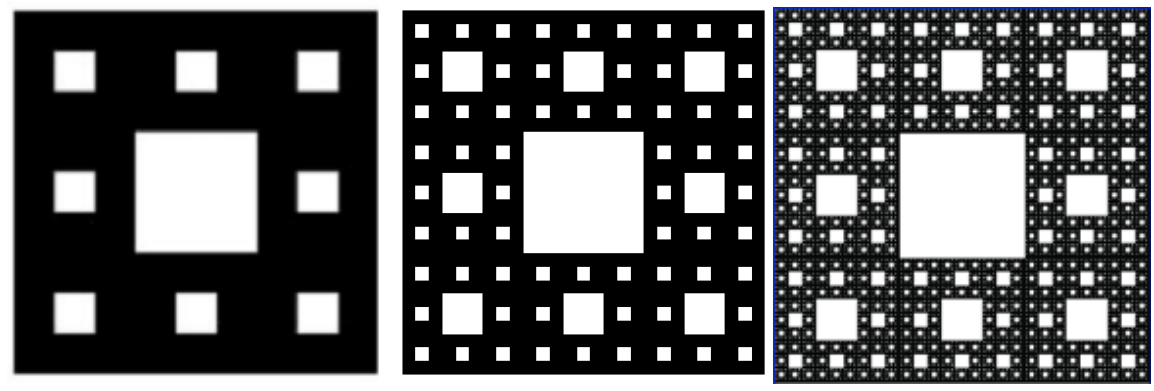

Figure 6. $A_{3}^{2}(3)$ (Sierpinski carpet) in its second, third, and fourth iterations

Figure 7 shows the $A_{4}^{2}(4)$ map of Figure 5(b) after the second iteration.

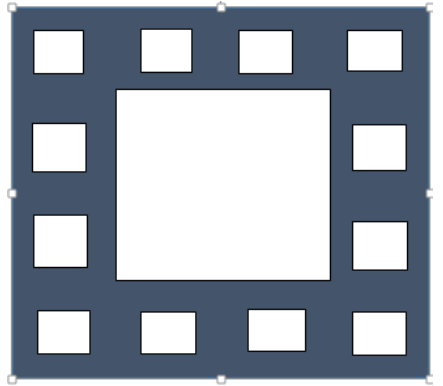

This fractal is basically similar to the Sierpinski carpet excepting that the whites are bigger.

\section{Related symmetric checkerboard maps}

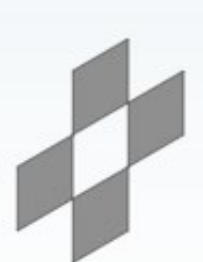

(a)

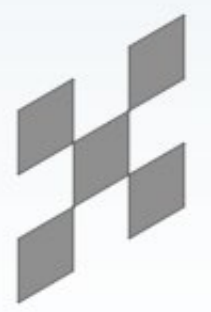

(b)

Figure $8.3 \times 3$ Checkerboard, $C$, together with complement, $\bar{C}$ 


$$
\begin{gathered}
d[C(3)]=\frac{\ln 4}{\ln 3} \cong 1.262 \\
d[\bar{C}]=\frac{\ln 5}{\ln 3} \cong 1.465
\end{gathered}
$$

A mapping where $\mathrm{C}$ is rotated will naturally not change the dimensionality.

The next order symmetric checkerboard map will be $5 \times 5$ as shown in Figure 9:

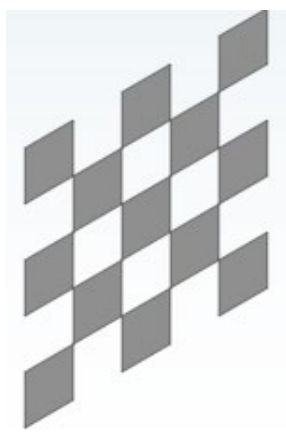

Figure $9.5 \times 5$ Checkerboard map

Its dimensionality will be given by

$$
d[C(5)]=\frac{\ln 13}{\ln 5} \cong 1.594
$$

Its complement mapping (with three white squares at the top that is not shown here) will have dimensionality of $\frac{\ln 12}{\ln 5} \cong 1.544$. Since the difference in the counts of black and white squares will only be one, the two dimensionality figures will be quite close to each other.

One can also modify the checkerboard map in a variety of ways to get new fractals.

\section{Annular fractals in three dimensions}

The Menger sponge, the 3-dimensional generalization of $A_{3}^{2}(3)$, is very well known [21]. 
Let us consider the generalization of $A_{4}^{3}(4)$. In its first iteration it will look like Figure 10.

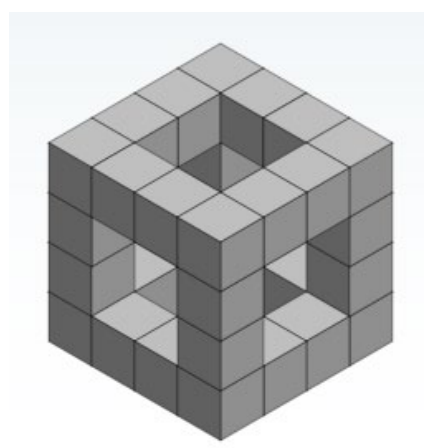

Figure 10. $A_{4}^{3}(4)$ mapping in three-dimensions

The number of sub-cubes generated from a cube at each iteration is 32 and therefore

$$
d\left[A_{4}^{3}(4)\right]=\frac{\ln 32}{\ln 4} \cong 2.5
$$

The first iteration of $A_{5}^{3}(5)$ is shown in Figure 11.

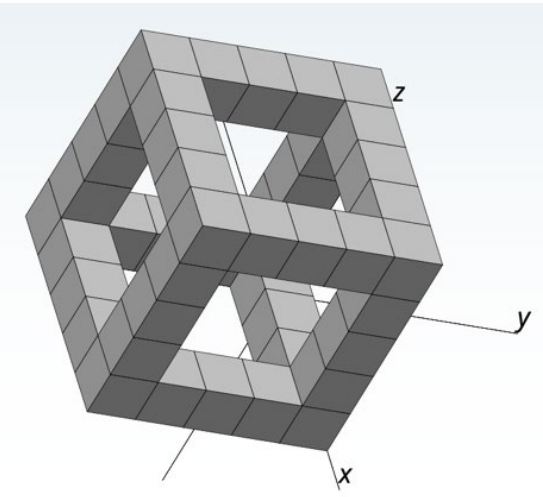

Figure 11. $A_{5}^{3}(5)$ mapping

Its dimensionality is:

$$
d\left[A_{5}^{3}(5)\right]=\frac{\ln 44}{\ln 5} \cong 2.351
$$

This dimensionality value is less than that for $A_{4}^{3}(4)$ for it is less dense.

A much more dense fractal $A_{5}^{3}(1)$ is given in Figure 12 . 


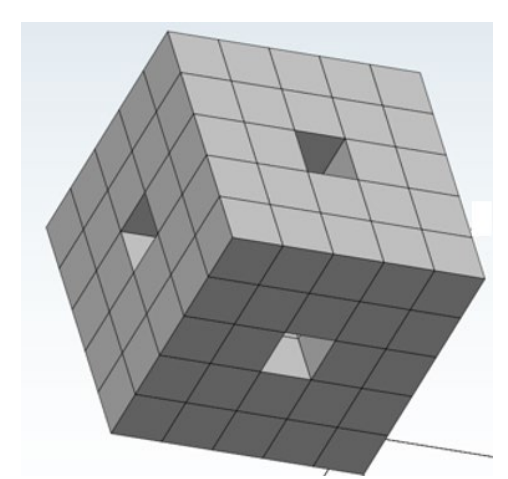

Figure 12. $A_{5}^{3}(1)$ mapping

$$
d\left[A_{5}^{3}(1)\right]=\frac{\ln 112}{\ln 5} \cong 2.932
$$

Finally, we present $A_{5}^{3}(5,1)$ mapping in Figure 13.

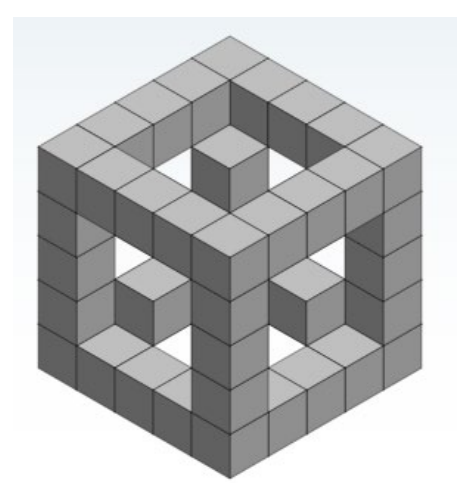

Figure 13. $A_{5}^{3}(5,1)$ mapping

It is more dense than $A_{5}^{3}(5)$ and less dense than $A_{5}^{3}(1)$ mapping.

$$
d\left[A_{5}^{3}(5,1)\right]=\frac{\ln 57}{\ln 5} \cong 2.512
$$

\section{Conclusions}

The paper introduced new fractal families with regular, square annular and checkerboard structures. The complementary mappings were defined and a notation to represent the families was proposed.

This work may be extended by defining other classes that are either larger in the number of sub-squares at each iteration or are according to some other simple definition for the basic planar forms. It would be worthwhile to look for members of these families in engineered and natural physical systems. 


\section{REFERENCES}

1. Mandelbrot, B. B., The Fractal Geometry of Nature. W. H. Freeman (1983)

2. Falconer, K.J., Fractal Geometry: Mathematical Foundations and Applications. (Wiley, 2003)

3. Kak, S. Power series models of self-similarity in social networks. Information Sciences, 376, 31-38 (2017)

4. Kak, S. Variations on the Newcomb-Benford law. (2018). arXiv:1806.06695

5. Miller, S. J., ed. Benford's Law: Theory and Applications. Princeton University Press (2015)

6. Bunde, A., Havlin, S., Fractals in Science. Springer (2013)

7. Vicsek, T. Fluctuations and scaling in biology. Oxford University Press (2001)

8. Burns, T. Rajan, R. A mathematical approach to correlating objective spectrotemporal features of non-linguistic sounds with their subjective perceptions in humans. Frontiers in Neuroscience. 13: 794 (2019)

9. Kak, S. Fractals with optimum information dimension. Circuits Syst. Signal Process. 40 (2021); https://link.springer.com/article/10.1007/s00034-021-01726-5

10. Kak, S. The base-e representation of numbers and the power law. Circuits Syst. Signal Process. 40, 490-500 (2021)

11. Kak, S. Information, representation, and structure. International Conference on Recent Trends in Mathematics and Its Applications to Graphs, Networks and Petri Nets, New Delhi, India (2020)

12. Kak, S. The intrinsic dimensionality of data. Circuits Syst. Signal Process. 40, 2599$2607(2021)$

13. Stillinger, F.H., Axiomatic basis for spaces with noninteger dimensions. Journal of Mathematical Physics 18, 1224- 1234 (1977)

14. Tarasov, V.E. Vector calculus in non-integer dimensional space and its applications to fractal media. Communications in Nonlinear Science and Numerical Simulation 20, 360-374 (2015)

15. Kak, S. Information theory and dimensionality of space. Scientific Reports 10, 20733 (2020).

16. Kak, S. Asymptotic freedom in noninteger spaces. Scientific Reports 11, 1-5 (2021)

17. Jun, L., Ostoja-Starzewski, M. Edges of Saturn's rings are fractal. SpringerPlus 4, 158 (2015)

18. Kak, S. Information-theoretic view of the variation of the gravitational constant. (2021). https://www.techrxiv.org/articles/preprint/Informationtheoretic_View_of_the_Variation_of_the_Gravitational_Constant/14527104

19. Edgar, G. Measure, Topology, and Fractal Geometry. New York: Springer-Verlag (2008)

20. Iwaniec, T., Martin, G. Geometric Function Theory and Non-linear Analysis, Oxford Mathematical Monographs (2001)

21. Semmes, S. Some Novel Types of Fractal Geometry. Oxford Mathematical Monographs (2001) 